Article

\title{
An Elastodiffusive Orthotropic Euler-Bernoulli Beam Considering Diffusion Flux Relaxation
}

\author{
Dmitry Tarlakovskii ${ }^{1,2}$ and Andrei Zemskov ${ }^{1,2, *}$ \\ 1 Research Institute of Mechanics, Lomonosov Moscow State University, 119192 Moscow, Russia; \\ tdvhome@mail.ru \\ 2 Moscow Aviation Institute (National Research University), 125993 Moscow, Russia \\ * Correspondence: azemskov1975@mail.ru; Tel.: +7-926-522-38-24
}

Received: 29 December 2018; Accepted: 30 January 2019; Published: 6 February 2019

\begin{abstract}
This article considers an unsteady elastic diffusion model of Euler-Bernoulli beam oscillations in the presence of diffusion flux relaxation. We used the model of coupled elastic diffusion for a homogeneous orthotropic multicomponent continuum to formulate the problem. A model of unsteady bending for the elastic diffusive Euler-Bernoulli beam was obtained using Hamilton's variational principle. The Laplace transform on time and the Fourier series expansion by the spatial coordinate were used to solve the obtained problem.
\end{abstract}

Keywords: elastic diffusion; coupled problem; unsteady problem; Green's function; integral transformation; multicomponent continuum; Euler-Bernoulli beam

\section{Introduction}

In order to solve various types of fundamental, applied, and technical problems, scientists and engineers often have to consider the unsteady interaction between various physical fields. An example of this is the interaction between mechanical and diffusion fields. Among the recent publications, it can be noted that [1-19] were devoted to this problem. In particular, [1-11] considered the thermal effects, while the electromagnetic effects were studied in [12-15]. In addition, the analysis of fast unsteady processes in relatively short time periods requires the relaxation of thermal and diffusion perturbations to be taken into account $[1,2,4-6,8,9,11-13]$.

Based on the reviewed publications, it can be concluded that numerical-analytical methods constructed on the Laplace and Fourier integral transformations are used to solve unsteady mechanodiffusion problems. In this case, the Durbin method is mainly used for the Laplace transform inversion. This method allows the Mellin integral to be expressed through the Fourier transform. Special numerical algorithms are used to do the inverse Fourier transform. Descriptions of these algorithms can be found in [20-22].

However, there is no universal algorithm for the inversion of Laplace transform, as noted in [23]. Each specific algorithm fits a certain class of functions. At the same time, the specificity of Laplace images influences the choice of suitable systems of functions with which one can approximate the unknown original values. In addition, Laplace images are very cumbersome to obtain by solving the coupled problems. It is not always possible in practice to verify the capability of using one or the other algorithm to find Laplace transform originals. Thus, it can be concluded that the Laplace transform inversion is the main mathematical complexity that arises when solving unsteady problems (mechanodiffusion problems in particular).

This article considers an unsteady elastic diffusion problem of Euler-Bernoulli beam oscillations in the presence of diffusion flux relaxation. It also proposes a method to construct a solution based on the use of the integral Laplace transform and expansion into series by eigenfunctions. 
The expansion coefficients are represented as rational functions, which simplifies the issue of inverse Laplace transform. Thus, the problem considered in the article is solved analytically.

It should be noted that analogues of the considered problem are absent in well-known scientific publications.

\section{Problem Formulation}

We consider the unsteady oscillation problem of elastic diffusion Euler-Bernoulli beam in the presence of diffusion flux relaxation. The beam in general formulation is under the action of longitudinal and transverse forces. Also, bending moments are at its ends. The load scheme is presented in Figure 1.

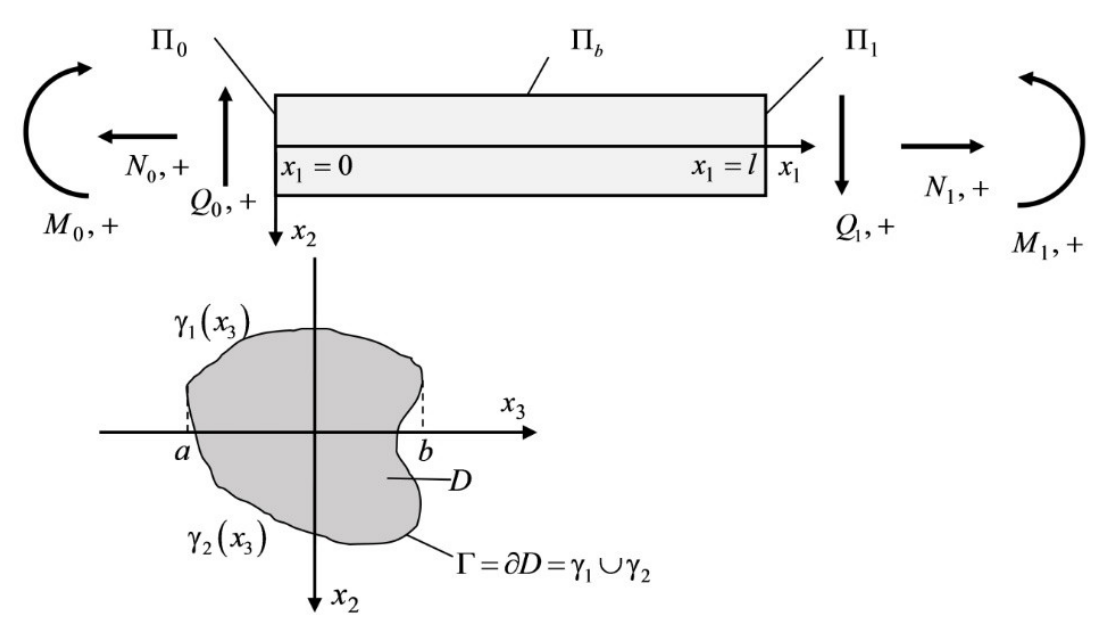

Figure 1. The formulation of the problem.

For the mathematical problem formulation, we use the coupled elastic diffusion continuum model in a rectangular Cartesian coordinate system, which has the following form [1-18]:

$$
\ddot{u}_{i}=\frac{\partial \sigma_{i j}}{\partial x_{j}}+F_{i}, \quad \dot{\eta}^{(q)}=-\frac{\partial J_{i}^{(q)}}{\partial x_{i}}+Y^{(q)} \quad(q=\overline{1, N}) .
$$

where the point denotes a time derivative; $\sigma_{i j}$ and $J_{i}^{(q)}$ are the stress tensor components and the diffusion flux vector, respectively. They are defined as follows (the beam material is perfect solid solution):

$$
\sigma_{i j}=C_{i j k l} \frac{\partial u_{k}}{\partial x_{l}}-\sum_{q=1}^{N} \alpha_{i j}^{(q)} \eta^{(q)}, \quad J_{i}^{(q)}+\tau^{(q)} \dot{j}_{i}^{(q)}=-D_{i j}^{(q)} \frac{\partial \eta^{(q)}}{\partial x_{j}}+\Lambda_{i j k l}^{(q)} \frac{\partial^{2} u_{k}}{\partial x_{j} \partial x_{l}} \quad(q=\overline{1, N}) .
$$

Here, the dots denote the time derivative. All quantities in (1) and (2) are dimensionless. For them, the following notations are used:

$$
\begin{gathered}
x_{i}=\frac{x_{i}^{*}}{l}, \quad u_{i}=\frac{u_{i}^{*}}{l}, \quad \tau=\frac{C t}{l}, \quad C_{i j k l}=\frac{C_{i j k l}^{*}}{C_{1111}}, \quad C^{2}=\frac{C_{1111}^{*}}{\rho}, \quad \alpha_{i j}^{(q)}=\frac{\alpha_{i j}^{*(q)}}{C_{1111}} \\
\tau_{q}=\frac{C \tau^{(q)}}{L}, \quad D_{i j}^{(q)}=\frac{D_{i j}^{*(q)}}{C l}, \quad \Lambda_{i j k l}^{(q)}=\frac{m^{(q)} D_{i j}^{*(q)} \alpha_{k l}^{*(q)} n_{0}^{(q)}}{\rho R T_{0} C l}, \quad F_{i}=\frac{\rho l F_{i}^{*}}{S_{1111}}, \quad Y^{(q)}=\frac{l Y^{*(q)}}{C},
\end{gathered}
$$

where $t$ is time; $x_{i}^{*}$ are rectangular Cartesian coordinates; $\rho$ is the medium density; $u_{i}^{*}$ are displacement vector components; $C_{i j k l}^{*}$ are elastic constant tensor components; $T_{0}$ is the initial temperature; $D_{i j}^{*(q)}$ are the self-diffusion coefficients; $R$ is the universal gas constant; $m^{(q)}$ is the molar mass; $\eta^{(q)}=n^{(q)}-n_{0}^{(q)}$ 
is the concentration increment of $q$-th component in the $N$-component medium; $n_{0}^{(q)}$ and $n^{(q)}$ are the initial and actual concentrations (mass fractions); $\alpha_{i j}^{*(q)}$ are coefficients characterizing the medium volumetric changes due to diffusion; $l$ is beam length; $F_{i}$ and $Y^{(q)}$ are mechanical and diffusive bulk perturbations; and $\tau^{(q)}$ is the relaxation time of diffusion perturbations.

The formulation of the problem is completed by the initial and boundary conditions.

Initial conditions:

$$
\left.u_{i}\right|_{\tau=t_{0}}=u_{i 0},\left.\quad \dot{u}_{i}\right|_{\tau=t_{0}}=v_{i 0},\left.\quad \eta^{(q)}\right|_{\tau=t_{0}}=\eta_{0}^{(q)} \quad(q=\overline{1, N}) .
$$

Here, $u_{i 0}, v_{i 0}, \eta_{0}^{(q)}$ are the given functions of spatial coordinates. Further in the paper, we assume that $t_{0}=0, \quad u_{i 0}=0, \quad v_{i 0}=0, \quad \eta_{0}^{(q)}=0$.

Boundary conditions (domain $G$ is bounded; $n_{i}$ are components of the outer normal unit vector to $\left.\partial G, \partial G=\Pi_{u} \cup \Pi_{\sigma}=\Pi_{\eta} \cup \Pi_{J}\right):$

$$
\left.u_{i}\right|_{\Pi_{u}}=U_{i},\left.\quad \sigma_{i j} n_{j}\right|_{\Pi_{\sigma}}=P_{i},\left.\quad \eta^{(q)}\right|_{\Pi_{\eta}}=N^{(q)},\left.\quad\left(J_{i}^{(q)}+\tau_{q} \dot{J}_{i}^{(q)}\right)\right|_{\Pi_{J}}=I_{i}^{(q)} \quad(\tau>0, q=\overline{1, N}) .
$$

The quantities on the boundary conditions on right sides are surface kinematic $U_{i}, N^{(q)}$ and dynamic $P_{i}, I_{i}^{(q)}$ perturbations.

To construct the beam bending equations, a transition to the variational formulation of the problem (1)-(5) is used. According to Hamilton's variational principle, the relations (1)-(5) can be regarded as a condition for the stationarity of a certain functional $H\left(u_{i}, \eta^{(q)}\right)$, whose variation is written thus:

$$
\begin{gathered}
\delta H=\int_{t_{1}}^{t_{2}} d \tau \int_{G}\left(\ddot{u}_{i}-\frac{\partial \sigma_{i j}}{\partial x_{j}}-F_{i}\right) \delta u_{i} d G+\sum_{q=1}^{N} \int_{t_{1}}^{t_{2}} d \tau \int_{G}\left(1+\tau_{q} \frac{\partial}{\partial \tau}\right)\left(\eta^{(q)}+\frac{\partial J_{i}^{(q)}}{\partial x_{i}}-Y^{(q)}\right) \delta \eta^{(q)} d G+ \\
+\int_{t_{1}}^{t_{2}} \iint_{\Pi_{\sigma}}\left(\sigma_{i j} n_{j}-P_{i}\right) \delta u_{i} d S d \tau+\sum_{q=1}^{N} \int_{t_{1}}^{t_{2}} \iint_{\Pi_{J}}\left(J_{i}^{(q)}+\tau_{q} J_{i}^{(q)}-I_{i}^{(q)}\right) n_{i} \delta \eta^{(q)} d S d \tau .
\end{gathered}
$$

Further, we will assume that

i The axis $\mathrm{Ox}_{3}$ is the central axis of the cross section. In this case,

$$
\iint_{D} x_{2} d x_{2} d x_{3}=0
$$

ii The side surface $\Pi_{b}$ is free from mechanical loads, i.e.,

$$
\left.\sigma_{i j} n_{j}\right|_{\Pi_{b}}=0
$$

iii We also assume that there is no mass transfer through the side surface,

$$
\left.\left(J_{i}^{(q)}+\tau_{q} \dot{J}_{i}^{(q)}\right)\right|_{\Pi_{b}}=0
$$

iv The beam material is a homogeneous ortotropic continuum.

The bending of beam is considered in plane $x_{1} O x_{2}$. Then $u_{k}=u_{k}\left(x_{1}, x_{2}, \tau\right), k=1,2 ; \quad u_{3}=0$; $\varepsilon_{i 3}=0$. Mass transfer occurs also in the plane $x_{1} O x_{2}$, i.e., $\eta^{(q)}=\eta^{(q)}\left(x_{1}, x_{2}, \tau\right)$. 
$\mathrm{v}$ Transverse deflections are considered small. Then, the linearization of the unknown quantities with respect to the variable $x_{2}$ will have the following form:

$$
\begin{gathered}
u_{1}\left(x_{1}, x_{2}, \tau\right)=u\left(x_{1}, \tau\right)-x_{2} \chi\left(x_{1}, \tau\right), \quad u_{2}\left(x_{1}, x_{2}, \tau\right)=v\left(x_{1}, \tau\right)+x_{2} \psi\left(x_{1}, \tau\right), \\
\eta^{(q)}\left(x_{1}, x_{2}, \tau\right)=N_{q}\left(x_{1}, \tau\right)+x_{2} H_{q}\left(x_{1}, \tau\right) .
\end{gathered}
$$

vi The cross sections after deformation remain normal to the neutral line of the beam (Euler-Bernoulli 's beam theory). Also, we assume that there are no deformations along the $O x_{2}$ axis $[24,25]$.

$$
\varepsilon_{22}=\frac{\partial u_{2}}{\partial x_{2}}=\psi=0 \Rightarrow \psi=0, \quad \chi\left(x_{1}, \tau\right)=v^{\prime}\left(x_{1}, \tau\right) .
$$

Then, Equation (10) will take the following form:

$$
u_{1}=u-x_{2} v^{\prime}, \quad u_{2}=v, \quad u_{3}=0, \quad u=u\left(x_{1}, \tau\right), \quad v=v\left(x_{1}, \tau\right) .
$$

Here, the prime denotes the derivative with respect to the variable $x_{1}$.

From (8) and (9), it follows that,

$$
\begin{gathered}
\left.\sigma_{22} n_{2}\right|_{\Pi_{b}}=\left.\sigma_{22} n_{2}\right|_{\Gamma}=\left.\sigma_{22}\right|_{\gamma_{2}\left(x_{3}\right)} ^{\gamma_{1}\left(x_{3}\right)}=0 \\
\int_{G} \frac{\partial \sigma_{22}}{\partial x_{2}} d G=\int_{0}^{1} d x_{1} \iint_{D} \frac{\partial \sigma_{22}}{\partial x_{2}} d x_{2} d x_{3}=\int_{0}^{1} d x_{1} \int_{a}^{b} \int_{\gamma_{2}\left(x_{3}\right)}^{\gamma_{1}\left(x_{3}\right)} \frac{\partial \sigma_{22}}{\partial x_{2}} d x_{2} d x_{3}=\left.\int_{0}^{1} d x_{1} \int_{a}^{b} \sigma_{22}\right|_{\gamma_{2}\left(x_{3}\right)} ^{\gamma_{1}\left(x_{3}\right)} d x_{3}=0 .
\end{gathered}
$$

The components of the stress tensor and the diffusion flow vector will have the following form:

$$
\begin{gathered}
\sigma_{11}=C_{11} \frac{\partial u_{1}}{\partial x_{1}}+C_{12} \frac{\partial u_{2}}{\partial x_{2}}-\sum_{q=1}^{N} \alpha_{1}^{(q)} \eta^{(q)}=\left(u^{\prime}-x_{2} v^{\prime \prime}\right)-\sum_{q=1}^{N} \alpha_{1}^{(q)}\left(N_{q}+x_{2} H_{q}\right), \quad \sigma_{12}=C_{66}\left(\frac{\partial u_{1}}{\partial x_{2}}+\frac{\partial u_{2}}{\partial x_{1}}\right)=0 \\
\sigma_{22}=C_{12} \frac{\partial u_{1}}{\partial x_{1}}+C_{22} \frac{\partial u_{2}}{\partial x_{2}}-\sum_{q=1}^{N} \alpha_{2}^{(q)} \eta^{(q)}=C_{12}\left(u^{\prime}-x_{2} v^{\prime \prime}\right)-\sum_{q=1}^{N} \alpha_{2}^{(q)}\left(N_{q}+x_{2} H_{q}\right), \\
J_{1}^{(q)}+\tau_{q} \dot{J}_{1}^{(q)}=-D_{1}^{(q)} \frac{\partial \eta^{(q)}}{\partial x_{1}}+\Lambda_{11}^{(q)} \frac{\partial^{2} u_{1}}{\partial x_{1}^{2}}+\Lambda_{12}^{(q)} \frac{\partial^{2} u_{2}}{\partial x_{1} \partial x_{2}}=-D_{1}^{(q)}\left(N_{q}^{\prime}+x_{2} H_{q}^{\prime}\right)+\Lambda_{11}^{(q)}\left(u^{\prime \prime}-x_{2} v^{\prime \prime \prime}\right), \\
J_{2}^{(q)}+\tau_{q} \dot{J}_{2}^{(q)}=-D_{2}^{(q)} \frac{\partial \eta}{\partial x_{2}}+\Lambda_{22}^{(q)} \frac{\partial^{2} u_{2}}{\partial x_{2}^{2}}+\Lambda_{21}^{(q)} \frac{\partial^{2} u_{1}}{\partial x_{1} \partial x_{2}}=-D_{2}^{(q)} H_{q}-\Lambda_{21}^{(q)} v^{\prime \prime} \quad(q=\overline{1, N}),
\end{gathered}
$$

where

$$
\begin{aligned}
& C_{11}=C_{1111}, \quad C_{12}=C_{1122}, \quad C_{22}=C_{2222}, \quad C_{66}=C_{1212}, \quad \alpha_{1}^{(q)}=\alpha_{11}^{(q)}, \quad \alpha_{2}^{(q)}=\alpha_{22}^{(q)} ， \\
& D_{1}^{(q)}=D_{11}^{(q)}, \quad D_{2}^{(q)}=D_{22}^{(q)}, \quad \Lambda_{11}^{(q)}=\Lambda_{1111}^{(q)}, \quad \Lambda_{12}^{(q)}=\Lambda_{1122}^{(q)}, \quad \Lambda_{21}^{(q)}=\Lambda_{2211}^{(q)}, \quad \Lambda_{22}^{(q)}=\Lambda_{2222}^{(q)} .
\end{aligned}
$$


Substituting the relations (7), (10)-(14) into (6), we obtain the following:

$$
\begin{aligned}
& \delta H=\int_{t_{1}}^{t_{2}} d \tau \int_{0}^{1}\left[F\left(\ddot{u}-u^{\prime \prime}+\sum_{q=1}^{N} \alpha_{1}^{(q)} N^{\prime}\right)-n\right] \delta u d x_{1}-\int_{t_{1}}^{t_{2}} d \tau \int_{0}^{1}\left[J_{3}\left(\ddot{v}^{\prime \prime}-v^{I V}-\sum_{q=1}^{N} \alpha_{1}^{(q)} H_{q}^{\prime \prime}\right)+m^{\prime}\right] \delta v d x_{1}+ \\
& +\int_{t_{1}}^{t_{2}} d \tau \int_{0}^{1}(F \ddot{v}-q) \delta v d x_{1}+\sum_{q=1}^{N} \int_{t_{1}}^{t_{2}} d \tau \int_{0}^{1}\left[F\left(N_{q}+\tau^{(q)} \ddot{N}_{q}-D_{1}^{(q)} N_{q}^{\prime \prime}+\Lambda_{11}^{(q)} u^{\prime \prime \prime}\right)-y^{(q)}\right] \delta N_{q} d x_{1}+ \\
& +\sum_{q=1}^{N} \int_{t_{1}}^{t_{2}} d \tau \int_{0}^{1}\left[J_{3}\left(H_{q}+\tau^{(q)} \ddot{H}_{q}-D_{1}^{(q)} H_{q}^{\prime \prime}-\Lambda_{11}^{(q)} v^{I V}\right)-z^{(q)}\right] \delta H_{q} d x_{1}+ \\
& \quad+\left.\int_{t_{1}}^{t_{2}}\left[J_{3}\left(\ddot{v}^{\prime}-v^{\prime \prime \prime}-\sum_{q=1}^{N} \alpha_{1}^{(q)} H_{q}^{\prime}\right)+m\right] \delta v\right|_{x_{1}=0} ^{x_{1}=1} d \tau+ \\
& +\left.\int_{t_{1}}^{t_{2}}\left\{\left[F\left(u^{\prime}-\sum_{q=1}^{N} \alpha_{1}^{(q)} N_{q}\right)-N\right] \delta u+\left[J_{3}\left(v^{\prime \prime}+\sum_{q=1}^{N} \alpha_{1}^{(q)} H_{q}\right)+M\right] \delta v^{\prime}-Q \delta v\right\}\right|_{x_{1}=0} ^{x_{1}=1} d \tau+ \\
& +\left.\sum_{q=1}^{N} \int_{t_{1}}^{t_{2}}\left\{\left[F\left(-D_{1}^{(q)} N_{q}^{\prime}+\Lambda_{11}^{(q)} u^{\prime \prime}\right)-\Gamma^{(q)}\right] \delta N_{q}-\left[J_{3}\left(D_{1}^{(q)} H_{q}^{\prime}+\Lambda_{11}^{(q)} v^{\prime \prime \prime}\right)+\Omega^{(q)}\right] \delta H_{q}\right\}\right|_{x_{1}=0} ^{x_{1}=1} d \tau .
\end{aligned}
$$

We introduce the following notation:

- $\quad \iint_{D} d x_{2} d x_{3}=F$ is the cross-sectional area,

- $\iint_{D} x_{2}^{2} d x_{2} d x_{3}=J_{3}$ is moment of inertia of the beam section relative to the axis $O x_{3}$,

- $\quad \iint_{D} F_{1} d x_{2} d x_{3}=n$ is the linearly distributed axial load,

- $\quad \iint_{D} F_{1} x_{2} d x_{2} d x_{3}=m$ is the linearly distributed moment,

- $\quad \iint_{D} F_{2} d x_{2} d x_{3}=q$ is the linearly distributed transverse load,

- $\quad \iint_{D}\left(Y^{(q)}+\tau^{(q)} Y^{(q)}\right) d x_{2} d x_{3}=y^{(q)}$ is the linear density of bulk mass transfer sources,

$$
\begin{gathered}
\iint_{D}\left(Y^{(q)}+\tau^{(q)} Y^{(q)}\right) x_{2} d x_{2} d x_{3}=z^{(q)}, \\
N\left(x_{1}\right)=\iint_{D} P_{1}\left(x_{1}, x_{2}, x_{3}, \tau\right) d x_{2} d x_{3}, \quad M\left(x_{1}\right)=\iint_{D} P_{1}\left(x_{1}, x_{2}, x_{3}, \tau\right) x_{2} d x_{2} d x_{3}, \quad Q\left(x_{1}\right)=\iint_{D} P_{2}\left(x_{1}, x_{2}, x_{3}, \tau\right) d x_{2} d x_{3}, \\
\Gamma^{(q)}\left(x_{1}\right)=\iint_{D} I_{1}^{(q)}\left(x_{1}, x_{2}, x_{3}, \tau\right) d x_{2} d x_{3}, \quad \Omega^{(q)}\left(x_{1}\right)=\iint_{D} x_{2} I_{1}^{(q)}\left(x_{1}, x_{2}, x_{3}, \tau\right) d x_{2} d x_{3} .
\end{gathered}
$$

Thus, the following boundary-value problems are the necessary conditions for the stationarity of the Hamilton functional considering (7)-(11):

$$
\begin{gathered}
\ddot{u}=u^{\prime \prime}-\sum_{q=1}^{N} \alpha_{1}^{(q)} N_{q}^{\prime}+\frac{n}{F}, \quad \dot{N}_{q}+\tau_{q} \ddot{N}_{q}=D_{1}^{(q)} N_{q}^{\prime \prime}-\Lambda_{11}^{(q)} u^{\prime \prime \prime}+\frac{y^{(q)}}{F} \\
\left.\left(u^{\prime}-\sum_{q=1}^{N} \alpha_{1}^{(q)} N_{q}\right)\right|_{x_{1}=0}=\frac{N_{0}}{F},\left.\quad\left(u^{\prime}-\sum_{q=1}^{N} \alpha_{1}^{(q)} N_{q}\right)\right|_{x_{1}=1}=\frac{N_{1}}{F}
\end{gathered}
$$




$$
\begin{gathered}
\left.\left(-D_{1}^{(q)} N_{q}^{\prime}+\Lambda_{11}^{(q)} u^{\prime \prime}\right)\right|_{x_{1}=0}=\frac{\Gamma_{0}^{(q)}}{F},\left.\quad\left(-D_{1}^{(q)} N_{q}^{\prime}+\Lambda_{11}^{(q)} u^{\prime \prime}\right)\right|_{x_{1}=1}=\frac{\Gamma_{1}^{(q)}}{F} ; \\
\ddot{v}^{\prime \prime}-\frac{F}{J_{3}} \ddot{v}=v^{I V}+\sum_{j=1}^{N} \alpha_{1}^{(q)} H_{j}^{\prime \prime}-\frac{q}{J_{3}}-\frac{m^{\prime}}{J_{3}}, \\
\dot{H}_{q}+\tau_{q} \ddot{H}_{q}=D_{1}^{(q)} H_{q}^{\prime \prime}+\Lambda_{11}^{(q)} v^{I V}+\frac{z^{(q)}}{J_{3}} ; \\
\left.\left(v^{\prime \prime}+\sum_{j=1}^{N} \alpha_{1}^{(q)} H_{j}\right)\right|_{x_{1}=0}=-\frac{M_{0}}{J_{3}},\left.\quad\left(v^{\prime \prime}+\sum_{j=1}^{N} \alpha_{1}^{(q)} H_{j}\right)\right|_{x_{1}=1}=-\frac{M_{1}}{J_{3}} ; \\
\left.\left(v^{\prime \prime \prime}+\sum_{j=1}^{N} \alpha_{1}^{(q)} H_{j}^{\prime}-\ddot{v}^{\prime}\right)\right|_{x_{1}=0}=\frac{Q_{0}+\left.m\right|_{x_{1}=0}}{J_{3}},\left.\quad\left(v^{\prime \prime \prime}+\sum_{j=1}^{N} \alpha_{1}^{(q)} H_{j}^{\prime}-\ddot{v}^{\prime}\right)\right|_{x_{1}=1}=\frac{Q_{1}+\left.m\right|_{x_{1}=1}}{J_{3}} ; \\
\left.\left(D_{1}^{(q)} H_{q}^{\prime}+\Lambda_{11}^{(q)} v^{\prime \prime \prime}\right)\right|_{x_{1}=0}=-\frac{\Omega_{0}^{(q)}}{J_{3}},\left.\quad\left(D_{1}^{(q)} H_{q}^{\prime}+\Lambda_{11}^{(q)} v^{\prime \prime \prime}\right)\right|_{x_{1}=1}=-\frac{\Omega_{1}^{(q)}}{J_{3}}
\end{gathered}
$$

where

$$
\begin{gathered}
N_{0}=N(0), \quad N_{1}=N(1), \quad M_{0}=M(0), \quad M_{1}=M(1), \quad Q_{0}=Q(0), \quad Q_{1}=Q(1), \\
\Gamma_{0}^{(q)}=\Gamma^{(q)}(0), \quad \Gamma_{1}^{(q)}=\Gamma^{(q)}(1), \quad \Omega_{0}^{(q)}=\Omega^{(q)}(0), \quad \Omega_{1}^{(q)}=\Omega^{(q)}(1) .
\end{gathered}
$$

In accordance with the variational principle of Lagrange, the boundary conditions (15)-(21) combined with the kinematic boundary conditions are as follows:

$$
\begin{array}{cc}
\left.u\right|_{x_{1}=0}=U_{0}, & \left.u\right|_{x_{1}=1}=U_{1} ; \\
\left.N_{q}\right|_{x_{1}=0}=N_{q 0}, & \left.N_{q}\right|_{x_{1}=1}=N_{q 1} ; \\
\left.v\right|_{x_{1}=0}=V_{0}, & \left.v\right|_{x_{1}=1}=V_{1} ; \\
\left.v^{\prime}\right|_{x_{1}=0}=V_{0}^{\prime}, & \left.v^{\prime}\right|_{x_{1}=1}=V_{1}^{\prime} ; \\
\left.H_{q}\right|_{x_{1}=0}=H_{q 0}, & \left.H_{q}\right|_{x_{1}=1}=H_{q 1} ; \\
\left.H_{q}^{\prime}\right|_{x_{1}=0}=H_{q 0}^{\prime},\left.\quad H_{q}^{\prime}\right|_{x_{1}=1}=H_{q 1}^{\prime} .
\end{array}
$$

Problems (15), (16), and (23) as well as (15), (17), and (22) are equivalent to one-dimensional problems of elastic diffusion for a layer. Their solutions are constructed using Laplace transform and decomposition into trigonometric Fourier series [26-28]. The papers [29,30] also considered problems with other combinations of boundary conditions that are solved by the method of equivalent boundary conditions.

Thus, the object of further consideration in this article will be initial boundary value problems for Equation (18) with a combination of boundary conditions (19), (20), and (24)-(27). Initial conditions will be assumed to be zero. 


\section{Method of Solution}

We consider the problems (18), (19), (24), (26). The solutions of the problem are represented in the form $(q=\overline{1, N+1})$ :

$$
\begin{gathered}
v(x, \tau)=\sum_{k=1}^{N+2} \int_{0}^{\tau}\left[G_{1 k}(x, \tau-t) f_{k 1}(t)+G_{1 k}(1-x, \tau-t) f_{k 2}(t)\right] d t+\sum_{k=1}^{N+1} \int_{0}^{\tau} \int_{0}^{1} \widetilde{G}_{1 k}(x, \xi, \tau-t) F_{k}(\xi, t) d \xi d t, \\
H_{q}(x, \tau)=\sum_{k=1}^{N+2} \int_{0}^{\tau}\left[G_{q+1, k}(x, \tau-t) f_{k 1}(t)+G_{q+1, k}(1-x, \tau-t) f_{k 2}(t)\right] d t+ \\
+\sum_{k=1}^{N+1} \int_{0}^{\tau} \int_{0}^{1} \widetilde{G}_{q+1, k}(x, \xi, \tau-t) F_{k}(\xi, t) d \xi d t
\end{gathered}
$$

Here, $x=x_{1} ; G_{m k}$ and $\widetilde{G}_{m k}$ are surface and bulk Green's functions; $F_{1}(x, \tau)=\left(q+m^{\prime}\right) / J_{3}$ and $F_{q+1}(x, \tau)=z^{(q)} / J_{3}$ are body forces entering into Equation (18); and $f_{k l}(t)$ are surface disturbances that have the following form:

$$
\begin{gathered}
f_{11}(\tau)=-\frac{M_{0}(\tau)}{J_{3}}, \quad f_{12}(\tau)=-\frac{M_{1}(\tau)}{J_{3}} \\
f_{21}(\tau)=V_{0}, \quad f_{22}(\tau)=V_{1}, \quad f_{q+2,1}(\tau)=H_{q 0}(\tau), \quad f_{q+2,2}(\tau)=H_{q 1}(\tau) .
\end{gathered}
$$

The surface Green's functions $G_{m k}$ satisfy

$$
\begin{gathered}
\ddot{G}_{1 k}^{\prime \prime}-\frac{F}{J_{3}} \ddot{G}_{1 k}=G_{1 k}^{I V}+\sum_{j=1}^{N} \alpha_{1}^{(j)} G_{j+1, k}^{\prime \prime} \\
\dot{G}_{q+1, k}+\tau_{q} \ddot{G}_{q+1, k}=D_{1}^{(q)} G_{q+1, k}^{\prime \prime}+\Lambda_{11}^{(q)} G_{1 k}^{I V}, \\
\left.\left(G_{1 k}^{\prime \prime}+\sum_{j=1}^{N} \alpha_{1}^{(j)} G_{j+1, k}\right)\right|_{x_{1}=0}=\delta_{1 k} \delta(\tau),\left.\quad\left(G_{1 k}^{\prime \prime}+\sum_{j=1}^{N} \alpha_{1}^{(j)} G_{j+1, k}\right)\right|_{x_{1}=1}=0 \\
\left.G_{1 k}\right|_{x_{1}=0}=\delta_{2 k} \delta(\tau),\left.\quad G_{1 k}\right|_{x_{1}=1}=0,\left.\quad G_{q+1, k}\right|_{x_{1}=0}=\delta_{q+2, k} \delta(\tau),\left.\quad G_{q+1, k}\right|_{x_{1}=1}=0 .
\end{gathered}
$$

The bulk Green's functions $\widetilde{G}_{m k}$ satisfy

$$
\begin{gathered}
\ddot{\widetilde{G}}_{1 k}^{\prime \prime}-\frac{F}{J_{3}} \ddot{\widetilde{G}}_{1 k}=\widetilde{G}_{1 k}^{I V}+\sum_{j=1}^{N} \alpha_{1}^{(j)} \widetilde{G}_{j+1, k}^{\prime \prime}-\delta_{1 k} \delta(x-\xi) \delta(\tau), \\
\dot{\widetilde{G}}_{q+1, k}+\tau_{q} \ddot{\widetilde{G}}_{q+1, k}=D_{1}^{(q)} \widetilde{G}_{q+1, k}^{\prime \prime}+\Lambda_{11}^{(q)} \widetilde{G}_{1 k}^{I V}+\delta_{q+1, k} \delta(x-\xi) \delta(\tau),
\end{gathered}
$$

and homogeneous boundary conditions corresponding to (30). 
We consider the problem of finding the surface Green's functions $G_{m k}$. Applying the Laplace transformation with respect to time to (29) and (30), we get the following ( $s$ is the Laplace transform parameter):

$$
\begin{gathered}
s^{2} G_{1 k}^{L^{\prime \prime}}(x, s)-\frac{F}{J_{3}} s^{2} G_{1 k}^{L}(x, s)=\left[G_{1 k}^{I V}(x, s)\right]^{L}+\sum_{j=1}^{N} \alpha_{1}^{(j)} G_{j+1, k}^{L^{\prime \prime}}(x, s), \\
\left(s+\tau_{q} s^{2}\right) G_{q+1, k}^{L}(x, s)=D_{1}^{(q)} G_{q+1, k}^{L^{\prime \prime}}(x, s)+\Lambda_{11}^{(q)}\left[G_{1 k}^{I V}(x, s)\right]^{L}, \\
\left.\left(G_{1 k}^{L \prime \prime}+\sum_{j=1}^{N} \alpha_{j} G_{j+1, k}^{L}\right)\right|_{x_{1}=0}=\delta_{1 k},\left.\quad\left(G_{1 k}^{L^{\prime \prime}}+\sum_{j=1}^{N} \alpha_{j} G_{j+1, k}^{L}\right)\right|_{x_{1}=1}=0, \\
\left.G_{1 k}^{L}\right|_{x_{1}=0}=\delta_{2 k},\left.\quad G_{1 k}^{L}\right|_{x_{1}=1}=0,\left.\quad G_{q+1, k}^{L}\right|_{x_{1}=0}=\delta_{q+1, k},\left.\quad G_{q+1, k}^{L}\right|_{x_{1}=1}=0 .
\end{gathered}
$$

Next, we multiply each equation by $\sin \lambda_{n} x$ and integrate in the interval $[0,1]$. The result is as follows:

$$
\begin{gathered}
k_{1}\left(\lambda_{n}, s\right) G_{1 k}^{L s}\left(\lambda_{n}, s\right)-\lambda_{n}^{2} \sum_{j=1}^{N} \alpha_{1}^{(j)} G_{j+1, k}^{L s}\left(\lambda_{n}, s\right)=F_{1 k}\left(\lambda_{n}\right), \\
-\lambda_{n}^{4} \Lambda_{11}^{(q)} G_{1 k}^{L s}\left(\lambda_{n}, s\right)+k_{q+1}\left(\lambda_{n}, s\right) G_{q+1, k}^{L s}\left(\lambda_{n}, s\right)=F_{q+1, k}\left(\lambda_{n}\right),
\end{gathered}
$$

where

$$
\begin{gathered}
k_{1}\left(\lambda_{n}, s\right)=\left(\lambda_{n}^{2}+\frac{F}{J_{3}}\right) s^{2}+\lambda_{n}^{4}, \quad F_{q+1, k}\left(\lambda_{n}\right)=2 \lambda_{n}\left(\Lambda_{11}^{(q)} \delta_{1 k}-\Lambda_{11}^{(q)} \lambda_{n}^{2} \delta_{2 k}+D_{1}^{(q)} \delta_{q+2, k}-\Lambda_{11}^{(q)} \sum_{j=1}^{N} \alpha_{1}^{(j)} \delta_{j+2, k}\right), \\
k_{q+1}\left(\lambda_{n}, s\right)=s+\tau_{q} s^{2}+D_{1}^{(q)} \lambda_{n}^{2}, \quad F_{1 k}\left(\lambda_{n}\right)=-2 \lambda_{n} \delta_{1 k}+2 \lambda_{n}\left(s^{2}+\lambda_{n}^{2}\right) \delta_{2 k} \\
G_{m k}^{L}(x, s)=\sum_{n=1}^{\infty} G_{m k}^{L s}\left(\lambda_{n}, s\right) \sin \lambda_{n} x, \quad G_{m k}^{L s}\left(\lambda_{n}, s\right)=2 \int_{0}^{1} G_{m k}^{L}(x, s) \sin \lambda_{n} x d x, \quad \lambda_{n}=\pi n .
\end{gathered}
$$

The solution of system (31) has the following form:

$$
\begin{gathered}
G_{1 k}^{L s}\left(\lambda_{n}, s\right)=\frac{P_{1 k}\left(\lambda_{n}, s\right)}{P\left(\lambda_{n}, s\right)}, \quad G_{q+1,1}^{L s}\left(\lambda_{n}, s\right)=\frac{2 \lambda_{n} \Lambda_{11}^{(q)}}{k_{q+1}\left(\lambda_{n}, s\right)}+\frac{P_{q+1,1}\left(\lambda_{n}, s\right)}{Q_{q}\left(\lambda_{n}, s\right)}, \\
G_{q+1,2}^{L s}\left(\lambda_{n}, s\right)=-\frac{2 \Lambda_{11}^{(q)} \lambda_{n}^{3}}{k_{q+1}\left(\lambda_{n}, s\right)}+\frac{P_{q+1,2}\left(\lambda_{n}, s\right)}{Q_{q}\left(\lambda_{n}, s\right)} \\
G_{q+1, p+2}^{L s}\left(\lambda_{n}, s\right)=\frac{2 \lambda_{n}\left(D_{1}^{(q)} \delta_{q p}-\Lambda_{11}^{(q)} \alpha_{p}\right)}{k_{q+1}\left(\lambda_{n}, s\right)}+\frac{P_{q+1, p+2}\left(\lambda_{n}, s\right)}{Q_{q}\left(\lambda_{n}, s\right)} \quad(q, p=\overline{1, N}, \quad k=\overline{1, N+1}) .
\end{gathered}
$$


Here,

$$
\begin{gathered}
P\left(\lambda_{n}, s\right)=k_{1}\left(\lambda_{n}, s\right) \Pi\left(\lambda_{n}, s\right)-\lambda_{n}^{6} \sum_{j=1}^{N} \Lambda_{11}^{(j)} \alpha_{1}^{(j)} \Pi_{j}\left(\lambda_{n}, s\right), \quad Q_{q}\left(\lambda_{n}, s\right)=k_{q+1}\left(\lambda_{n}, s\right) P\left(\lambda_{n}, s\right), \\
P_{11}\left(\lambda_{n}, s\right)=-2 \lambda_{n}\left[\Pi\left(\lambda_{n}, s\right)-\lambda_{n}^{2} \sum_{j=1}^{N} \alpha_{1}^{(j)} \Lambda_{11}^{(j)} \Pi_{j}\left(\lambda_{n}, s\right)\right], \\
P_{12}\left(\lambda_{n}, s\right)=2 \lambda_{n}\left[\left(s^{2}+\lambda_{n}^{2}\right) \Pi\left(\lambda_{n}, s\right)-\lambda_{n}^{4} \sum_{j=1}^{N} \alpha_{1}^{(j)} \Lambda_{11}^{(j)} \Pi_{j}\left(\lambda_{n}, s\right)\right], \\
P_{1, q+2}\left(\lambda_{n}, s\right)=2 \alpha_{1}^{(q)} \lambda_{n}^{3}\left[D_{1}^{(q)} \Pi_{q}\left(\lambda_{n}, s\right)-\sum_{j=1}^{N} \alpha_{1}^{(j)} \Lambda_{11}^{(j)} \Pi_{j}\left(\lambda_{n}, s\right)\right], \\
P_{q+1,1}\left(\lambda_{n}, s\right)=2 \lambda_{n}^{5} \Lambda_{11}^{(q)}\left[\Pi\left(\lambda_{n}, s\right)-\lambda_{n}^{2} \sum_{j=1}^{N} \alpha_{1}^{(j)} \Lambda_{11}^{(j)} \Pi_{j}\left(\lambda_{n}, s\right)\right], \\
P_{q+1,2}\left(\lambda_{n}, s\right)=2 \lambda_{n}^{5} \Lambda_{11}^{(q)}\left[\left(s^{2}+\lambda_{n}^{2}\right) \Pi\left(\lambda_{n}, s\right)-\lambda_{n}^{4} \sum_{j=1}^{N} \alpha_{1}^{(j)} \Lambda_{11}^{(j)} \Pi_{j}\left(\lambda_{n}, s\right)\right], \\
P_{q+1, p+2}\left(\lambda_{n}, s\right)=2 \alpha_{1}^{(p)} \lambda_{n}^{7} \Lambda_{11}^{(q)}\left[D_{1}^{(p)} \Pi_{p}\left(\lambda_{n}, s\right)-\sum_{j=1}^{N} \alpha_{1}^{(j)} \Lambda_{11}^{(j)} \Pi_{j}\left(\lambda_{n}, s\right)\right], \\
\Pi\left(\lambda_{n}, s\right)=\prod_{j=1}^{N} k_{j+1}\left(\lambda_{n}, s\right), \quad \Pi_{j}\left(\lambda_{n}, s\right)=\prod_{r=1, r \neq j}^{N} k_{r+1}\left(\lambda_{n}, s\right) .
\end{gathered}
$$

The Laplace originals of functions in (32) have the following form [28]:

$$
\begin{gathered}
G_{1 k}^{s}\left(\lambda_{n}, \tau\right)=\sum_{j=1}^{2 N+2} A_{1 k}^{(j)}\left(\lambda_{n}\right) e^{s_{j}\left(\lambda_{n}\right) \tau}, \quad \xi_{1,2}\left(\lambda_{n}\right)=\frac{-1 \pm \sqrt{1-4 \tau_{q} D_{1}^{(q)} \lambda_{n}^{2}}}{2 \tau_{q}}, \\
G_{q+1,1}^{s}\left(\lambda_{n}, \tau\right)=\sum_{l=1}^{2}\left[\frac{2 \Lambda_{11}^{(q)} \lambda_{n}}{1+2 \tau_{q} \xi_{l}\left(\lambda_{n}\right)}+A_{q+1,1}^{(2 N+2+l)}\left(\lambda_{n}\right)\right] e^{\xi_{l}\left(\lambda_{n}\right) \tau}+\sum_{j=1}^{2 N+2} A_{q+1,1}^{(j)}\left(\lambda_{n}\right) e^{s_{j}\left(\lambda_{n}\right) \tau}, \\
G_{q+1,2}^{s}\left(\lambda_{n}, \tau\right)=\sum_{l=1}^{2}\left[\frac{-2 \Lambda_{11}^{(q)} \lambda_{n}^{3}}{1+2 \tau_{q} \xi_{l}\left(\lambda_{n}\right)}+A_{q+1,2}^{(2 N+2+l)}\left(\lambda_{n}\right)\right] e^{\xi_{l}\left(\lambda_{n}\right) \tau}+\sum_{j=1}^{2 N+2} A_{q+1,2}^{(j)}\left(\lambda_{n}\right) e^{s_{j}\left(\lambda_{n}\right) \tau}, \\
G_{q+1, p+2}^{s}\left(\lambda_{n}, \tau\right)=\sum_{l=1}^{2}\left[\frac{2\left(D_{1}^{(q)} \delta_{q p}-\Lambda_{11}^{(q)} \alpha_{1}^{(p)}\right) \lambda_{n}}{1+2 \tau_{q} \xi_{l}\left(\lambda_{n}\right)}+A_{q+1, p+2}^{(2 N+2+l)}\left(\lambda_{n}\right)\right] e^{\xi_{l}\left(\lambda_{n}\right) \tau}+\sum_{j=1}^{2 N+2} A_{q+1, p+2}^{(j)}\left(\lambda_{n}\right) e^{s_{j}\left(\lambda_{n}\right) \tau}, \\
A_{1 k}^{(j)}\left(\lambda_{n}\right)=\frac{P_{1 k}\left(\lambda_{n}, s_{j}\left(\lambda_{n}\right)\right)}{P^{\prime}\left(\lambda_{n}, s_{j}\left(\lambda_{n}\right)\right)}(j=\overline{1, N+3}, \quad k=\overline{1, N+2,} \quad r=\overline{1, N+4}, \quad q, p=\overline{1, N),} \\
A_{q+1, k}^{(r)}\left(\lambda_{n}\right)=\frac{P_{q+1, k}\left(\lambda_{n}, s_{r}\left(\lambda_{n}\right)\right)}{Q_{q}^{\prime}\left(\lambda_{n}, s_{r}\left(\lambda_{n}\right)\right)}, \quad A_{q+1, k}^{(2 N+2+l)}=\frac{P_{q+1, k}\left(\lambda_{n}, \xi_{l}\left(\lambda_{n}\right)\right)}{Q_{q}^{\prime}\left(\lambda_{n}, \xi_{l}\left(\lambda_{n}\right)\right)} \quad(l=1,2),
\end{gathered}
$$

where $s_{j}\left(\lambda_{n}\right), j=\overline{1,2 N+2}$ are zeros of polynomial $P\left(\lambda_{n}, s\right)$.

\section{Examples}

For the calculation example, we take the one-component $(N=1)$ aluminum medium with the following characteristics [31]:

$$
\begin{gathered}
C_{1111}^{*}=12.05 \cdot 10^{10} \frac{\mathrm{N}}{\mathrm{m}^{2}}, \quad T_{0}=800 \mathrm{~K}, \quad \rho=2700 \frac{\mathrm{kg}}{\mathrm{m}^{3}}, \\
\alpha_{11}^{*(1)}=4.2 \cdot 10^{5} \frac{\mathrm{J}}{\mathrm{mol}}, \quad D_{11}^{*(1)}=7.73 \cdot 10^{-14} \frac{\mathrm{m}^{2}}{\mathrm{~s}}, \quad L=0.1 \mathrm{~m}, \quad \tau^{(q)}=200 \mathrm{~s} .
\end{gathered}
$$


We assume that the beam has a rectangular section with height $h=0.1 \mathrm{~m}$ and width $b=0.05 \mathrm{~m}$. The geometrical characteristics of the section is as follows:

$$
F=b h=5.00 \cdot 10^{-5} \mathrm{~m}^{2}, \quad J_{3}=\frac{b h^{3}}{12}=4.17 \cdot 10^{-10} \mathrm{~m}^{4} .
$$

Example 1. We assume in the boundary conditions (19), (24), and (26):

$$
\begin{gathered}
f_{11}(\tau)=-\frac{M_{0}(\tau)}{J_{3}}=H(\tau), \quad f_{12}(\tau)=-\frac{M_{1}(\tau)}{J_{3}}=H(\tau), \\
f_{21}(\tau)=V_{0}(\tau)=0, \quad f_{22}(\tau)=V_{1}(\tau)=0, \quad f_{31}(\tau)=H_{20}(\tau)=0, \quad f_{32}(\tau)=H_{21}(\tau)=0 .
\end{gathered}
$$

Then, according to (28), in the absence case of volume perturbations, we have the following:

$$
\begin{gathered}
v(x, \tau)=\int_{0}^{\tau}\left[G_{11}(x, \tau-t)+G_{11}(1-x, \tau-t)\right] H(t) d t= \\
=2 \sum_{n=1}^{\infty} \sin \frac{\lambda_{n}}{2} \cos \lambda_{n}\left(\frac{1}{2}-x\right) \sum_{j=1}^{4} A_{11}^{(j)}\left(\lambda_{n}\right) \frac{e^{s_{j}\left(\lambda_{n}\right) \tau}-1}{s_{j}\left(\lambda_{n}\right)} \\
H_{1}(x, \tau)=\int_{0}^{\tau}\left[G_{21}(x, \tau-t)+G_{21}(1-x, \tau-t)\right] H(t) d t= \\
=2 \sum_{n=1}^{\infty} \sin \frac{\lambda_{n}}{2} \cos \lambda_{n}\left(\frac{1}{2}-x\right) \sum_{l=1}^{2}\left(\frac{2 \Lambda_{11}^{(1)} \lambda_{n}}{1+2 \tau_{\eta} \xi_{l}\left(\lambda_{n}\right)}+A_{21}^{(4+l)}\left(\lambda_{n}\right)\right) \frac{e^{\xi_{l}}\left(\lambda_{n}\right) \tau}{\xi_{l}\left(\lambda_{n}\right)}+ \\
+2 \sum_{n=1}^{\infty} \sin \frac{\lambda_{n}}{2} \cos \lambda_{n}\left(\frac{1}{2}-x\right) \sum_{j=1}^{4} A_{21}^{(j)}\left(\lambda_{n}\right) \frac{e^{s_{j}}\left(\lambda_{n}\right) \tau}{s_{j}\left(\lambda_{n}\right)} .
\end{gathered}
$$

The calculation results are shown in Figure 2. It should be noted that the effects associated with the diffusion flux relaxation for given perturbations (33) do not appear at all. The graphs for the beam deflection $v(x, \tau)$ and the concentration increment $H_{1}(x, \tau)$ without considering the relaxation have the same view as in Figure 2.

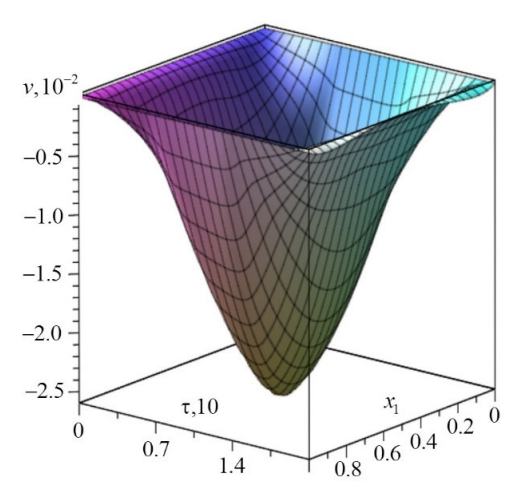

(a)

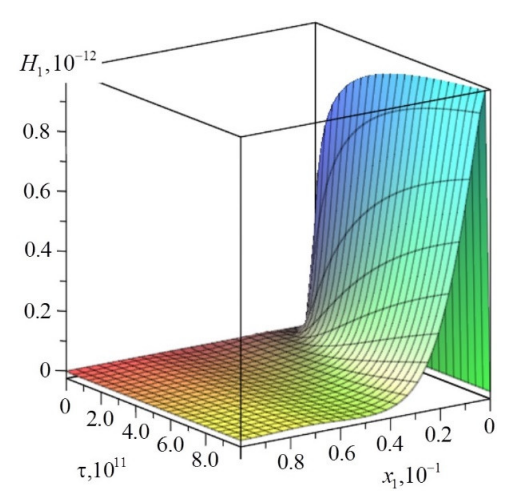

(b)

Figure 2. The results of calculations using Equation (34): (a) The beam deflections $v(x, \tau)$; (b) The concentration increment $H_{1}(x, \tau)$.

Example 2. Now, we assume in the boundary conditions (19), (24), and (26) that

$$
\begin{gathered}
f_{11}(\tau)=-\frac{M_{0}(\tau)}{J_{3}}=0, \quad f_{12}(\tau)=-\frac{M_{1}(\tau)}{J_{3}}=0, \\
f_{21}(\tau)=V_{0}(\tau)=0, \quad f_{22}(\tau)=V_{1}(\tau)=0, \quad f_{31}(\tau)=H_{20}(\tau)=H(\tau), \quad f_{32}(\tau)=H_{21}(\tau)=H(\tau) .
\end{gathered}
$$


That (28)

$$
\begin{gathered}
v(x, \tau)=\int_{0}^{\tau}\left[G_{13}(x, \tau-t)+G_{13}(1-x, \tau-t)\right] H(t) d t= \\
=2 \sum_{n=1}^{\infty} \sin \frac{\lambda_{n}}{2} \cos \lambda_{n}\left(\frac{1}{2}-x\right) \sum_{j=1}^{4} A_{13}^{(j)}\left(\lambda_{n}\right) \frac{e^{s_{j}\left(\lambda_{n}\right) \tau}-1}{s_{j}\left(\lambda_{n}\right)} \\
H_{1}(x, \tau)=\int_{0}^{\tau}\left[G_{22}(x, \tau-t)+G_{22}(1-x, \tau-t)\right] H(t) d t= \\
=2 \sum_{n=1}^{\infty} \sin \frac{\lambda_{n}}{2} \cos \lambda_{n}\left(\frac{1}{2}-x\right) \sum_{l=1}^{2}\left(\frac{2\left(D_{1}^{(1)}-\alpha_{1}^{(1)} \Lambda_{11}^{(1)}\right) \lambda_{n}}{1+2 \tau_{q} \xi_{l}\left(\lambda_{n}\right)}+A_{23}^{(4+l)}\left(\lambda_{n}\right)\right) \frac{e^{\xi_{l}\left(\lambda_{n}\right) \tau}-1}{\xi_{l}\left(\lambda_{n}\right)}+ \\
+2 \sum_{n=1}^{\infty} \sin \frac{\lambda_{n}}{2} \cos \lambda_{n}\left(\frac{1}{2}-x\right) \sum_{j=1}^{4} A_{23}^{(j)}\left(\lambda_{n}\right) \frac{e^{s_{j}\left(\lambda_{n}\right) \tau}-1}{s_{j}\left(\lambda_{n}\right)} .
\end{gathered}
$$

The calculation results are shown in Figure 3.

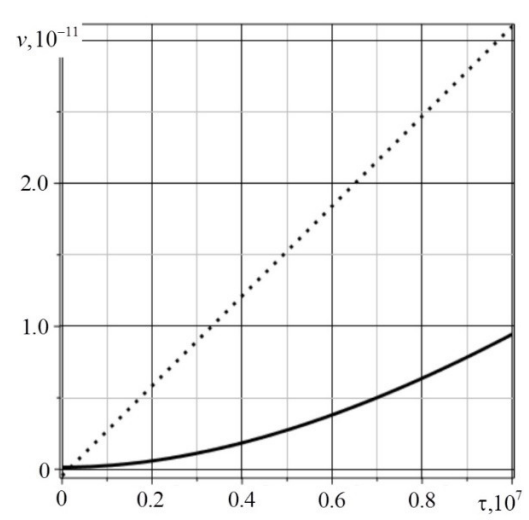

(a)

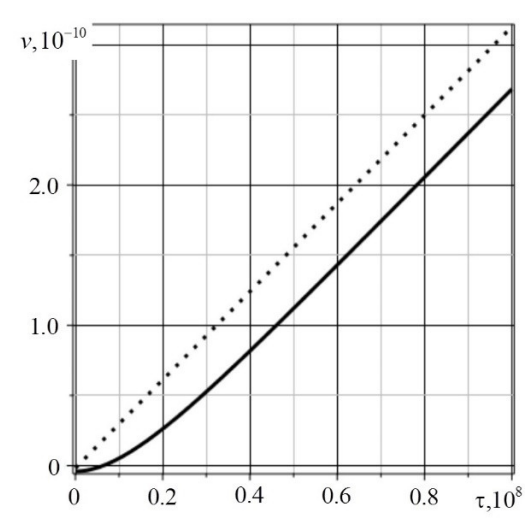

(b)

Figure 3. The beam deflections $v(x, \tau), x=0.5$. The solid line is the solution with relaxation of diffusion fluxes, and the dashed line is the solution without relaxation of diffusion fluxes: (a) Effect of diffusion processes relaxation on the beam deflections at $\tau \sim 10^{7}$; (b) Effect of diffusion processes relaxation on the beam deflections at $\tau \sim 10^{8}$.

Relaxation effects are manifested under the condition $\tau \leq \tau_{q}$ (dimensionless time $10^{7}$ is corresponds to $150 \mathrm{~s}$ ), as can be seen in Figure 3. The relaxation effects fade out with increasing time, which is demonstrated in Figure 4. A similar situation is observed for the concentration increment $H_{q}(x, \tau)$. Based on the completed study, it can be concluded that the relaxation effects manifestation essentially depends on both the material properties and the type of specified perturbations. The results obtained in this article are consistent with the results obtained by other researchers, for example, $[6,12,32,33]$. 


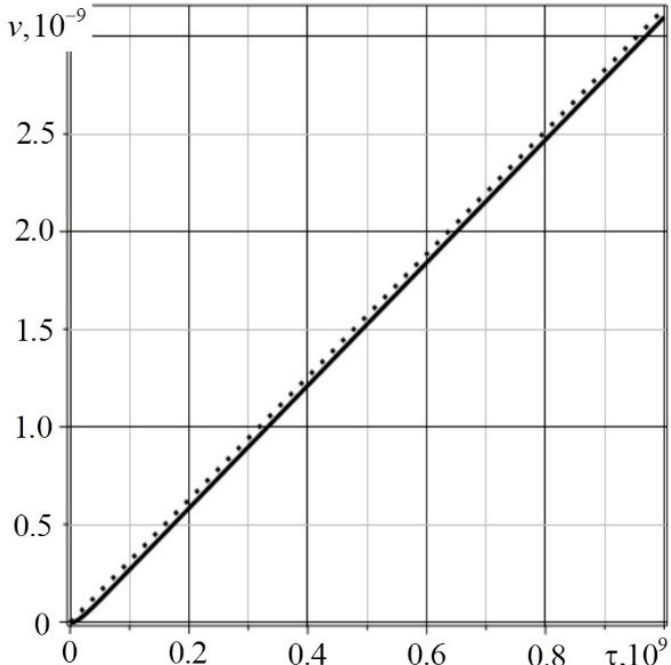

(a)

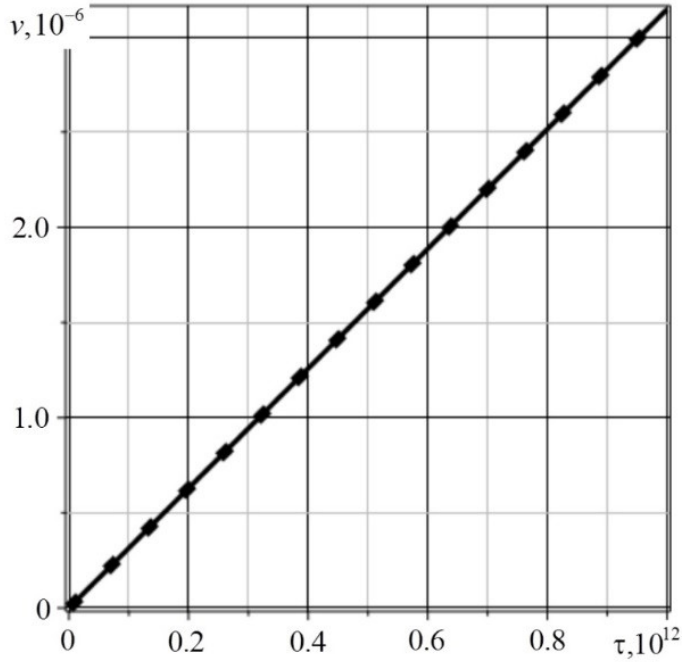

(b)

Figure 4. The beam deflections $v(x, \tau), x=0.5$. The solid line is the solution with relaxation of diffusion fluxes, and the dashed line is the solution without relaxation of diffusion fluxes: (a) Effect of diffusion processes relaxation on the beam deflections at $\tau \sim 10^{9}$; (b) Effect of diffusion processes relaxation on the beam deflections at $\tau \sim 10^{12}$.

\section{Conclusions}

The coupled unsteady oscillations model of an elastic diffusion Euler-Bernoulli beam was constructed using Hamilton's variational principle. An algorithm for constructing surface Green's functions was proposed. The use of the unknown function expansion into series by eigenfunctions allowed us to solve the problem associated with the Laplace transform inversion. Such an approach made it possible to find an analytical solution to the oscillation problem of an elastic diffusion Euler-Bernoulli beam.

On the basis of the developed model, the interaction of mechanical and diffusion fields was investigated. The influence of diffusion relaxation effects on the beam was analyzed. A number of test calculations showed that relaxation effects appeared only at the initial moments of time and under certain boundary conditions. The results of the calculations are presented in analytical and graphical forms.

Author Contributions: D.T. and A.Z. contributed equally to the writing of the main manuscript and preparation of the figures. Both authors reviewed the manuscript. Both authors contributed equally to the paper.

Funding: This work was funded by a subsidy from RFBR (Project 17-08-00663 A).

Conflicts of Interest: The funders had no role in the design of the study; in the collection, analyses, or interpretation of data; in the writing of the manuscript; or in the decision to publish the results.

\section{References}

1. Afram, A.Y.; Khader, S.E. 2D Problem for a Half-Space under the Theory of Fractional Thermoelastic Diffusion. Am. J. Sci. Ind. Res. 2014, 6, 47-57.

2. Atwa, S.Y.; Egypt, Z. Generalized Thermoelastic Diffusion with Effect of Fractional Parameter on Plane Waves Temperature-Dependent Elastic Medium. J. Mater. Chem. Eng. 2013, 1, 55-74.

3. Belova, I.V.; Murch, G.E. Thermal and diffusion-induced stresses in crystalline solids. J. Appl. Phys. 1995, 77, 127-134. [CrossRef]

4. Choudhary, S.; Deswal, S. Mechanical Loads on a Generalized Thermoelastic Medium with Diffusion. Meccanica 2010, 45, 401-413. [CrossRef] 
5. Elhagary, M.A. Generalized thermoelastic diffusion problem for an infinitely long hollow cylinder for short times. Acta Mech. 2011, 218, 205-215. [CrossRef]

6. El-Sayed, A.M. A two-dimensional generalized thermoelastic diffusion problem for a half-space. Math. Mech. Solids 2016, 21, 1045-1060. [CrossRef]

7. Knyazeva, A.G. Model of medium with diffusion and internal surfaces and some applied problems. Mater. Phys. Mech. 2004, 7, 29-36.

8. Kumar, R.; Chawla, V. Green's Functions in Orthotropic Thermoelastic Diffusion Media. Eng. Anal. Bound. Elem. 2012, 36, 1272-1277. [CrossRef]

9. Olesiak, Z.S.; Pyryev, Yu.A. A coupled quasi-stationary problem of thermodiffusion for an elastic cylinder. Int. J. Eng. Sci. 1995, 33, 773-780. [CrossRef]

10. Pidstryhach, Y.S. Differential equations of the problem of thermodiffusion in a solid deformable isotropic body. Dop. Akad. Nauk USSR 1961, 2, 169-172.

11. Sherief, H.H.; El-Maghraby, N.M. A Thick Plate Problem in the Theory of Generalized Thermoelastic Diffusion. Int. J. Thermophys. 2009, 30, 2044-2057. [CrossRef]

12. Aouadi, M. Variable electrical and thermal conductivity in the theory of generalized thermoelastic diffusion. Z. Angew. Math. Phys. 2005, 57, 350-366. [CrossRef]

13. Deswal, S.; Kalkal, K. A two-dimensional generalized electro-magneto-thermoviscoelastic problem for a half-space with diffusion. Int. J. Therm. Sci. 2011, 50, 749-759. [CrossRef]

14. Tarlakovskii, D.V.; Vestyak, V.A.; Zemskov, A.V. Dynamic Processes in Thermoelectromagnetoelastic and Thermoelastodiffusive Media. In Encyclopedia of Thermal Stress; Springer: London, UK, 2014; Volume 2, pp. 1064-1071.

15. Zhang, J.; Li, Y. A Two-Dimensional Generalized Electromagnetothermoelastic Diffusion Problem for a Rotating Half-Space. Hindawi Publ. Corp. Math. Probl. Eng. 2014, 2014, 1-12. [CrossRef]

16. Chu, J.L.; Lee, S. Diffusion-induced stresses in a long bar of square cross section. J. Appl. Phys. 1993, 73, 3211-3219. [CrossRef]

17. Freidin, A.B.; Korolev, I.K.; Aleshchenko, S.P.; Vilchevskaya, E.N. Chemical affinity tensor and chemical reaction front propagation: Theory and FE-simulations. J. Fract. 2016, 202, 245-259. [CrossRef]

18. Hwang, C.C.; Chen, K.M.; Hsieh, J.Y. Diffusion-induced stresses in a long bar under an electric field. J. Phys. D Appl. Phys. 1994, 27, 2155-2162. [CrossRef]

19. Indeitsev, D.A.; Semenov, B.N.; Sterlin, M.D. The Phenomenon of Localization of Diffusion Process in a Dynamically Deformed Solid. Dokl. Phys. 2012, 57, 171-173. [CrossRef]

20. Crump, K. Numerical Inversion of Laplace Transforms Using a Fourier Series Approximation. J. Assoc. Comput. Mach. 1976, 23, 89-96. [CrossRef]

21. Durbin, F. Numerical inversion of Laplace transforms: An efficient improvement to Dubner and Abate's method. Comput. J. 1974, 17, 371-376. [CrossRef]

22. Honig, G.; Hirdes, U. A method for the numerical inversion of Laplace transforms. J. Comput. Appl. Math. 1984, 10, 113-132. [CrossRef]

23. Poroshina, N.I.; Ryabov, V.M. Methods for Laplace Transform Inversion; Vestnik of the St. Petersburg 239 University: Cambridge, UK, 2011; Volume 44, pp. 214-222.

24. Mikhailova, E.Yu.; Tarlakovskii, D.V.; Fedotenkov, G.V. Obshchaya Teoriya Uprugikh Obolochek; MAI: Moscow, Russia, 2018.

25. Gorshkov, A.G.; Medvedsky, A.L.; Rabinsky, L.N.; Tarlakovsky, D.V. Volny v Sploshnykh Sredakh; Fizmatlit: Moscow, Russia, 2004.

26. Davydov, S.A.; Zemskov, A.V.; Tarlakovskii, D.V. An Elastic Half-Space under the Action of One-Dimensional Time-Dependent Diffusion Perturbations. Lobachevskii J. Math. 2015, 36, 503-509. [CrossRef]

27. Davydov, S.A.; Vestyak, A.V.; Zemskov, A.V.; Tarlakovskii, D.V. Unsteady one-dimensional problem of thermoelastic diffusion for homogeneous multicomponent continuum with a plane boundaries. Uchenye Zapiski Kazanskogo Univ. Ser. Fiziko-Matematicheskie Nauki 2018, 160, 183-196.

28. Davydov, S.A.; Zemskov, A.V. Unsteady One-dimensional Perturbations in Multicomponent Thermoelastic Layer with Cross-diffusion Effect. J. Phys. Conf. Ser. 2018, 1129, 012009. [CrossRef]

29. Zemskov, A.V.; Tarlakovskiy, D.V. Method of the equivalent boundary conditions in the unsteady problem for elastic diffusion layer. Mater. Phys. Mech. 2015, 23, 36-41. 
30. Davydov, S.A.; Zemskov, A.V.; Igumnov, L.A.; Tarlakovskiy, D.V. Non-stationary model of mechanical diffusion for half-space with arbitrary boundary conditions. Mater. Phys. Mech. 2016, 28, 72-76.

31. Grigoriev, I.S.; Meylikhov, I.Z. Fizicheskiye Velichiny: Sprovochnik; Energoatomizdat: Moscow, Russia, 1991.

32. Polyanin, A.D.; Vyazmin, A.V. differential-difference heat-conduction and diffusion models and equations with a finite relaxation time. Theor. Found. Chem. Eng. 2013, 47, 217-224. [CrossRef]

33. Szekeres, A.; Fekete, B. Continuummechanics-Heat Conduction-Cognition. Period. Polytech. Mech. Eng. 2015, 59, 8-15. [CrossRef]

(C) 2019 by the authors. Licensee MDPI, Basel, Switzerland. This article is an open access article distributed under the terms and conditions of the Creative Commons Attribution (CC BY) license (http://creativecommons.org/licenses/by/4.0/). 